\title{
A CHARACTERISATION OF HILBERT SPACES VIA ORTHOGONALITY AND PROXIMINALITY
}

\author{
FATHI B. SAIDI
}

\begin{abstract}
In this paper we adopt the notion of orthogonality in Banach spaces introduced by the author in [6]. There, the author showed that in any two-dimensional subspace $F$ of $E$, every nonzero element admits at most one orthogonal direction. The problem of existence of such orthogonal direction was not addressed before. Our main purpose in this paper is the investigation of this problem in the case where $E$ is a real Banach space. As a result we obtain a characterisation of Hilbert spaces stating that, if in every two-dimensional subspace $F$ of $E$ every nonzero element admits an orthogonal direction, then $E$ is isometric to a Hilbert space. We conclude by presenting some open problems.
\end{abstract}

\section{INTRODUCTION}

Usually, the notion of orthogonality is associated with inner product spaces. Many extensions to Banach spaces have been introduced through the decades by various authors, for example, Birkhoff [1], Roberts [5], James [3], Singer [9], Khalil [4], and, more recently, in [6]. We adopt here the straightforward and simple extension of orthogonality introduced in [6]:

Definition 1: A finite or infinite sequence $\left(x_{n}\right)_{n \in S}$, in a real or complex Banach space $E$ is said to be orthogonal if

$$
\left\|\sum_{n \in S} a_{n} x_{n}\right\|=\left\|\sum_{n \in S}\left|a_{n}\right| x_{n}\right\|, \quad \text { for each } \sum_{n \in S} a_{n} x_{n} \in E
$$

where the $a_{n}$ 's are scalars. If, in addition, $\left\|x_{n}\right\|=1$ for all $n \in S$, then $\left(x_{n}\right)_{n \in S}$ is said to be orthonormal. We write $x \perp y$ if $x$ is orthogonal to $y$.

This concept of orthogonality was used in [6] to obtain a convenient and practical characterisation of compact operators in $L(G, E)$, where $G$ is a normed space, $E$ is a Banach space that admits an orthonormal Schauder basis, and $L(G, E)$ is the set of bounded linear operators from $G$ into $E$. This characterisation, which was previously

Received 1st September, 2004

Copyright Clearance Centre, Inc. Serial-fee code: 0004-9727/05 \$A2.00+0.00. 
known to hold when $G$ and $E$ are Hilbert spaces, says that the set of compact operators in $L(F, E)$ is the closure in $L(F, E)$ of the set of finite-rank operators.

Other applications of the above concept of orthogonality can be found in [7] and [8]. There the author obtains explicit characterisations of orthogonality in the spaces $l_{S}^{p}(C)$, where $C$ is the set of complex numbers, $S$ is a set of positive integers, and $p$ is a real number in $[1, \infty)$. When $p=2$, the usual characterisation of orthogonality in the Hilbert spaces $l_{S}^{2}(C)$ is recovered.

In $[6$, Corollary 1$]$, the author established the following uniqueness result showing that in a two-dimensional subspace $F$ of $E$, every nonzero element admits at most one orthogonal direction in $F$ :

PROPOSITION 1. Let $x$ and $y$ be two nonzero elements in $E$ satisfying $x \perp y$. Then we have

$$
\{z \in \operatorname{span}\{x, y\}: z \perp x\}=\operatorname{span}\{y\} .
$$

The problem of existence of an orthogonal direction to an element $x$ in a twodimensional subspace $F$ of $E$ has not been addressed before. Our main purpose in this paper is the investigation of this problem in the case where $E$ is a real Banach space. The question of existence in the complex case, in other words when $\operatorname{dim}(F)=2$ as a complex Banach space, is left as an open problem for further investigation. Therefore, we consider in this paper the following problem:

Problem 1. Let $E$ be a real Banach space. Is it true that in every two-dimensional subspace $F$ of $E$ each nonzero vector admits a nonzero orthogonal vector?

We start in Section 2 by showing that, in order for Problem 1 to have an affirmative answer, it is necessary that every proximinal subspace of $E$ be Chebyshev (Lemma 1). We then show that if every hyperplane of $E$ is proximinal (equivalently, every hyperplane is Chebyshev, by Lemma 1) then $E$ must be isometric to a Hilbert space in order for Problem 1 to have an affirmative answer (Lemma 2). Note that if every hyperplane of $E$ is proximinal then $E$ is reflexive, [9, Corollary 2.4, p. 99]. We finish by establishing our main result that shows that, when $\operatorname{dim}(E) \geqslant 3$, the answer to Problem 1 is affirmative if and only if $E$ is isometric to a Hilbert space (Theorem 1). The case where $E$ is real and $\operatorname{dim}(E)=2$ is still an open problem, Problem 2 in Section 3. More questions are raised in Section 3 and are left as open problems for further investigation.

\section{A Characterisation of Hilbert Spaces}

Throughout this paper we let $\mathbb{R}$ denote the set of real numbers. We start with the following definition:

Definition 2: A subset $G$ of a Banach space $E$ is said to be proximinal if for every $x \in E$ there exists $y \in G$ such that

$$
d(x, y)=\inf _{g \in G} d(x, g):=d(x, G),
$$


where $d(.,$.$) denotes the distance in E$. A point $y$ satisfying equation (2.1) is called a best approximation of $x$ from $G$. The set of best approximations from $G$ of a point $x \in E$ is denoted by $\pi_{G}(x)$. If every point $x \in E$ admits a unique best approximation from $G$, then $G$ is said to be Chebyshev and the best approximation of $x$ from $G$ is also denoted by $\pi_{G}(x)$.

We now show that if $E$ is a real Banach space with $\operatorname{dim}(E) \geqslant 3$ then, in order for Problem 1 to have an affirmative answer, it is necessary that every proximinal subspace of $E$ be Chebyshev. Indeed we have:

Lemma 1. Let $E$ be a real Banach space of (finite or infinite) dimension $\geqslant 2$. Suppose that in every two-dimensional subspace $F$ of $E$ each nonzero vector admits a nonzero orthogonal vector. Then every proximinal subspace of $E$ is Chebyshev.

Proof: Let $G$ be a proximinal subspace of $E$. If $G=E$ or $G=\{0\}$, then there is nothing to prove. Therefore suppose that $G \neq E,\{0\}$. If $G$ is not Chebyshev then there exists $x \in E \backslash G$ and there exist $g_{1}, g_{2} \in G$ such that

$$
g_{1}, g_{2} \in \pi_{G}(x), g_{1} \neq g_{2} .
$$

Let

$$
S:=\left(g_{1}+\operatorname{span}\left\{g_{2}-g_{1}\right\}\right) \cap \pi_{G}(x) .
$$

Then $S$ is a closed and bounded line-segment given by

$$
S=\left[g_{3}, g_{4}\right]:=\left\{g_{3}+\lambda\left(g_{4}-g_{3}\right): 0 \leqslant \lambda \leqslant 1\right\},
$$

for some $g_{3}, g_{4} \in G$. Let $\bar{x}, \bar{g}$, and $F$ be given by,

$$
\bar{x}:=x-\frac{g_{3}+g_{4}}{2}, \bar{g}:=\frac{g_{4}-g_{3}}{2} \text {, and } F:=\operatorname{span}\{\bar{x}, \bar{g}\} .
$$

Then

$$
\begin{aligned}
\pi_{G}(\bar{x}) \cap F & =\pi_{G}(\bar{x}) \cap \operatorname{span}\{\bar{g}\}=\left(\pi_{G}(x)-\frac{g_{3}+g_{4}}{2}\right) \cap \operatorname{span}\{\bar{g}\} \\
& =\pi_{G}(x) \cap\left(\frac{g_{3}+g_{4}}{2}+\operatorname{span}\{\bar{g}\}\right)-\frac{g_{3}+g_{4}}{2} \\
& =\pi_{G}(x) \cap\left(g_{1}+\operatorname{span}\left\{g_{2}-g_{1}\right\}\right)-\frac{g_{3}+g_{4}}{2} \\
& =S-\frac{g_{3}+g_{4}}{2}=[-\bar{g}, \bar{g}] .
\end{aligned}
$$

It follows that

$$
\|\bar{x}-t \bar{g}\|=\|\bar{x}\|,-1 \leqslant t \leqslant 1,
$$

and

$$
\|\bar{x}-t \bar{g}\|>\|\bar{x}\|,|t|>1
$$


Now, consider the element $y \in F$ defined by

$$
y:=\bar{x}-\frac{1}{2} \bar{g}
$$

To finish the proof of the lemma, we shall show that $y$ has no nonzero orthogonal vectors in $F$. This would contradict the assumptions of the lemma and, consequently, $G$ must be Chebyshev. First note that the set $\{\bar{x}, \bar{g}\}$ is linearly independent. If there exists $z(\neq 0) \in F, z:=\alpha \bar{x}+\beta \bar{g}$ for some scalars $\alpha$ and $\beta$, such that $y \perp z$ then

$$
\|y-t z\|=\|y+t z\|
$$

for all $t \in \mathbb{R}$. If $\alpha \neq 0$ (we can assume without loss of generality that $\alpha>0$ ), then, for every $t \in(-1 / \alpha, 1 / \alpha)$, we have

$$
(1-t \alpha)\left\|\bar{x}-\frac{(1 / 2)+t \beta}{(1-t \alpha)} \vec{g}\right\|=(1+t \alpha)\left\|\bar{x}-\frac{(1 / 2)-t \beta}{(1+t \alpha)} \bar{g}\right\| .
$$

Let $t_{1}:=((1 / 2)+t \beta) /(1-t \alpha)$ and $t_{2}:=((1 / 2)-t \beta) /(1+t \alpha)$ and choose $t \neq 0$ small enough so that $t_{1}, t_{2} \in[-1,1]$. Then, by equation $(2.2)$, we obtain that

$$
(1-t \alpha)\|\bar{x}\|=(1+t \alpha)\|\bar{x}\|
$$

which is not possible, since $\|\bar{x}\| \neq 0$. Therefore we must have $\alpha=0$ and, consequently, $z=\beta \bar{g}, \beta \neq 0$. By equation (2.4) we obtain that

$$
\left\|\bar{x}-\left(\frac{1}{2}+t \beta\right) \bar{g}\right\|=\left\|\bar{x}-\left(\frac{1}{2}-t \beta\right) \bar{g}\right\| .
$$

Letting $t=3 /(2 \beta)$, we obtain $\|\bar{x}-2 \bar{g}\|=\|\bar{x}+\bar{g}\|$, which is not possible in view of equations (2.2) and (2.3). Therefore $y$ has no nonzero orthogonal vector in $F$. The proof of the lemma is now complete.

Under the extra assumption that every hyperplane in $E$ is proximinal, we obtain the following characterisation:

LEMMA 2. Let $E$ be a real Banach space of (finite or infinite) dimension $\geqslant 3$. Suppose that in every two-dimensional subspace $F$ of $E$ each nonzero vector admits a nonzero orthogonal vector. Also, suppose that every hyperplane in $E$ is proximinal. Then $E$ is isometric to a Hilbert space.

Proof: Let $H$ be an arbitrary hyperplane in $E$ and let $x \in E \backslash H$ be fixed. Since $H$ is proximinal, we obtain, by Lemma 1 , that $H$ is Chebyshev. We claim that

$$
\left\|\pi_{H}(x)\right\| \leqslant\|x\|, x \in E .
$$

Indeed, let $h:=\pi_{H}(x)$. If $h=0$, then nothing to prove. So, suppose that $h \neq 0$ and let $F=\operatorname{span}\{x, h\}$. Then there exists $h^{\perp}(\neq 0) \in F$ such $h^{\perp} \perp h$. Clearly, the set $\left\{h, h^{\perp}\right\}$ is linearly independent. Therefore

$$
x=\alpha h+\beta h^{\perp}
$$


for some $\alpha, \beta \in \mathbb{R}$. This implies that

$$
\|x-\alpha h\|=\left\|\beta h^{\perp}\right\| \leqslant\left\|\beta h^{\perp}+(\alpha-1) h \mid=\right\| x-h \|,
$$

where the inequality follows from the fact that $h \perp h^{\perp}$, [6, Theorem 2]. It follows that $\alpha h \in \pi_{H}(x)=\{h\}$ and, consequently, $\alpha=1$. Therefore $(x-h) \perp h$. This implies, that

$$
\|h\| \leqslant\|h+(x-h)\|=\|x\|
$$

and, consequently, that equation (2.5) holds. By [9, Theorem 5.1, p. 247], we obtain that $E$ is isometric to a Hilbert space, which completes the proof of the lemma.

We are now ready to give our main result showing that, when $\operatorname{dim}(E) \geqslant 3$, Problem 1 has an affirmative answer if and only if $E$ is isometric to a Hilbert space. Indeed, we have:

THEOREM 1. Let $E$ be a real Banach space of (finite or infinite) dimension $\geqslant 3$. Suppose that in every two-dimensional subspace $F$ of $E$ each nonzero vector admits a nonzero orthogonal vector. Then $E$ is isometric to a Hilbert space.

Proof: Let $G$ be a 2-dimensional subspace of $E$ and let $G^{\prime}$ be a 3-dimensional subspace of $E$ containing $G$. Now, let $H$ be any hyperplane in $G^{\prime}$. Since $H$ is finite dimensional, it is proximinal in $G^{\prime}$. It follows, by Lemma 2, that $G^{\prime}$ is isometric to a Hilbert space, which implies that $G$ is isometric to a Hilbert space. Hence every 2dimensional subspace of $E$ is isometric to a Hilbert space and consequently, by [2, p. 17], $E$ is isometric to a Hilbert space. This completes the proof of the theorem.

Note that the condition that in every two-dimensional subspace $F$ of $E$ each nonzero vector admits a nonzero orthogonal vector is equivalent to the condition that $\operatorname{span} x+x^{\perp}=E$ for every $x \in E$, where

$$
x^{\perp}:=\{z \in E: z \perp x\} .
$$

Therefore we have the following corollary:

COROLlary 1. Let $E$ be a real Banach space of (finite or infinite) dimension $\geqslant 3$. Suppose that, for every $x \in E, \operatorname{span}\{x\}+x^{\perp}=E$. Then $E$ is isometric to a Hilbert space.

\section{OPEN PRoblems}

We start this section by noting that Theorem 1 was established only for the cases where $E$ is a real Banach space of dimension $\geqslant 3$. The case where $\operatorname{dim}(E)=2$ is still an open problem:

Problem 2. Let $E$ be a real Banach space satisfying $\operatorname{dim}(E)=2$. Is it true that, if each nonzero vector admits a nonzero orthogonal vector then $E$ is isometric to a Hilbert space? 